\title{
Atenção ao abortamento em instituições hospitalares da rede SUS de Fortaleza, Ceará
}

\section{Attention to abortion in hospitals of the SUS network in Fortaleza, Ceará}

\author{
Katherine Jeronimo Lima' (D), Francisco José Maia Pinto', Francisco Herlânio Costa Carvalho², \\ Cybelle Façanha Barreto Medeiros Linard', Fátima Café Ribeiro dos Santos', \\ Fiama Kécia Silveira Teófilo', Glaucilândia Pereira Nunes' \\ ${ }^{1}$ Programa de Pós-graduação em Saúde Coletiva, Universidade Estadual do Ceará (UFC) - Fortaleza (CE), Brasil. \\ ²Departamento de Saúde Comunitária, Universidade Federal do Ceará (UFC) - Fortaleza (CE), Brasil.
}

Como citar: Lima KJ, Pinto FJM, Carvalho FHC, Linard CFBM, Santos FCR, Teófilo FKS, et al. Atenção ao abortamento em instituições hospitalares da rede SUS de Fortaleza, Ceará. Cad Saúde Colet,2020;28(1):77-86. https://doi.org/10.1590/1414-462X202028010297

\section{Resumo}

Introdução: O aborto é uma questão relevante para a saúde pública, por sua significância entre as causas de morbidade e mortalidade materna. Objetivo: Descrever o perfil sociodemográfico e reprodutivo, bem como a assistência prestada e os custos da internação de mulheres em abortamento nos hospitais vinculados à rede SUS de Fortaleza, Ceará. Métodos: Trata-se de estudo transversal, descritivo, por meio de entrevista, prontuários e relatórios da Autorização das Internações Hospitalares. Resultados: Entre as 119 mulheres entrevistadas, 65\% tinham menos de 30 anos, 78,2\% eram não brancas e 54\% possuíam baixa renda; a prevalência de mulheres que buscaram mais de um hospital para atendimento foi de $47 \%$ e $72,3 \%$ buscaram o serviço de saúde em até cinco dias; concentração de $98,3 \%$ do procedimento cirúrgico curetagem para finalização do aborto. Conclusão: Percebeu-se a existência de pontos frágeis na atenção ao abortamento e a necessidade de discutir a temática aborto nas políticas públicas de saúde.

Palavras-chave: aborto; hospitalização; atenção à saúde.

\begin{abstract}
Background: Abortion is a relevant issue for public health, due to is significance among the causes of maternal and mortality. Objective: to describe the sociodemographic and reproductive profile, as well as the assistance provided and the costs of the hospitalization of women in abortion in the hospitals linked in the SUS network in Fortaleza, Ceará. Methods: this is a cross-sectional, descriptive study by means of interviews, medical records and reports of the Authorization of Hospitalizations. Results: among the 119 women interviewed, $65 \%$ were under 30 years of age, $78.2 \%$ were non-white and $54 \%$ had low income; the prevalence of women who sought more than one hospital for care was $47 \%$ and $72.3 \%$ of them sought health care within five days; concentration of $98.3 \%$ were submitted to a surgical procedure curettage for abortion termination. Conclusion: it was noticed the existence of fragile points in the attention to abortion and the need to discuss abortion thematic in public health policies.
\end{abstract}

Keywords: abortion; hospitalization; health care.

Este é um artigo publicado em acesso aberto (Open Access) sob a licença Creative Commons Attribution, que permite uso, distribuição e reprodução em qualquer meio, sem restrições desde que o trabalho original seja corretamente citado.

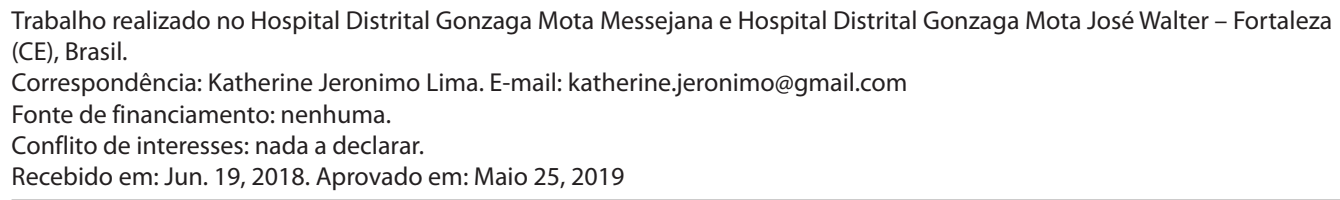




\section{INTRODUÇÃO}

No Brasil, o abortamento é considerado como a interrupção da gravidez sucedida antes da $22^{2}$ semana gestacional e tendo o concepto peso inferior a $500 \mathrm{~g}$. É classificado como grave problema de saúde pública, pois representa uma importante causa de morbimortalidade materna. Em 2016, representaram 8\% dos óbitos maternos em território nacional'. Na rede pública de saúde do Sistema Único de Saúde (SUS), ocorreram 205.075 internações por aborto em mulheres de 10 a 49 anos, em 2013. O Sudeste e Nordeste foram as regiões que se destacaram em maior número dessas internações ${ }^{2}$. No período de 2017, o Ceará esteve entre os cinco estados brasileiros com o maior número de internações por complicações abortivas, com a capital do estado, Fortaleza, na sexta posição ${ }^{3}$.

Outra situação, decorrente do abortamento, refere-se à curetagem, técnica cirúrgica para esvaziamento uterino, que representa o terceiro procedimento cirúrgico mais frequente nos estabelecimentos de saúde conveniados pelo SUS4.

Apesar dos altos índices, acredita-se que o contexto do aborto, no cenário brasileiro, seja bem pior, visto que os sistemas de informações oficiais, Sistema de Informações Hospitalares do Sistema Único de Saúde (SIH/SUS), retratam somente uma parcela das mulheres em situação abortiva, aquelas que necessitam de internação e que buscam o serviço público.

Além disso, existe a ilegalidade do aborto no país, pois é crime pelo Código Penal Brasileiro a interrupção voluntária da gravidez, quando provocado pela própria gestante ou terceiros. Porém, excetuam-se as seguintes circunstâncias: gravidez resultante de estupro, casos em que seja imprescindível para salvar a vida da mulher e fetos com anomalias congênitas, sem chance de sobrevivência extrauterina ${ }^{5,6}$.

Nos últimos anos, houve ampliação na agenda da saúde do Brasil de programas voltados à saúde da população feminina, dentre estes, às mulheres em situação de abortamento. O Ministério da Saúde elaborou a Norma Técnica para Atenção Humanizada ao Abortamento, como um guia sobre o manejo das situações abortivas, preconizando acolhimento, assistência de qualidade e humanização dos profissionais nos cuidados às mulheres em processo de aborto, independentemente do tipo de abortamento?.

Apesar disso, a atenção ao abortamento encontra-se distanciada das diretrizes propostas nacionalmente. Pesquisa realizada em três capitais nordestinas, que pretendia avaliar a qualidade da atenção ao abortamento em mulheres admitidas nos hospitais do SUS, apontou baixa avaliação na qualidade técnica, na continuidade do cuidado e apoio social, bem como verificou ausência de marcação de consultas de retorno para revisão, orientações sobre os riscos de uma gestação em curto tempo e sobre medidas contraceptivas. $O$ estudo também revelou que as unidades analisadas não eram estruturadas conforme as recomendações do modelo de atenção ao abortamento preconizado 8,9 .

Em face do contexto apresentado, este estudo objetivou descrever o perfil sociodemográfico e reprodutivo, bem como a assistência prestada e os custos de internação de mulheres em abortamento nos hospitais vinculados à rede SUS de Fortaleza, Ceará.

\section{MÉTODO}

Trata-se de uma pesquisa de corte transversal, com abordagem descritiva, realizada em duas unidades hospitalares públicas de referência, no município de Fortaleza, Ceará, no período de maio a outubro de 2017.

Os sujeitos desta pesquisa foram mulheres que buscaram os hospitais selecionados para atendimento à situação abortiva. Foram excluídas as menores de 18 anos, que não apresentaram condições clínicas e emocionais para participar da pesquisa bem como as que decidiram interromper a participação durante a entrevista. No final, somaram-se 119 participantes.

As variáveis estudadas foram organizadas em três grupos:

a) Sociodemográficas e reprodutivas: idade; cor/raça; escolaridade, renda familiar; número de abortos, diagnóstico de gravidez; gravidez planejada; uso de método contraceptivo;

b) Clínicas e assistência prestada, antes da internação: idade gestacional; sinais e/ou sintomas apresentados, local de busca para atendimento; internação no primeiro hospital de busca; 
número de hospitais percorridos; tempo de busca para atendimento (refere-se ao intervalo de tempo entre a apresentação dos sinais e sintomas e a procura para atendimento); tempo de espera para atendimento médico;

c) Assistência hospitalar, durante a internação e custos: procedimentos realizados (hemograma, urina tipo I, coagulograma, ultrassonografia antibioticoterapia, transfusão sanguínea); técnica de esvaziamento uterino; tempo decorrido entre a internação e esvaziamento uterino; dias de internação e valor pago.

Para a coleta de dados, as mulheres em situação abortiva eram identificadas através da listagem presente nos postos de enfermagem. Em seguida, dirigia-se ao leito, onde se realizava abordagem individualizada, e, depois do consentimento, seguia-se ao local reservado para entrevista, baseada em um formulário semiestruturado.

Posteriormente, foram utilizados prontuários para coletar dados sobre a assistência e também, a partir destes, obter o número da Autorização das Internações Hospitalares (AlH) respectivo para cada internamento. As informações contidas nos relatórios das AlH pagas foram usadas para aquisição de dados relacionados aos custos.

Após a coleta, os dados foram inseridos no programa da Microsoft Office Excel para construção do banco e analisados estatisticamente por meio do programa Statistical Package for the Social Scienses (SPSS), versão 18.0. Os dados foram analisados por meio de estatística descritiva: frequência, média e desvio padrão.

O estudo foi aprovado pelo Comitê de Ética e Pesquisa da Universidade Estadual do Ceará (CAAE: 66687817.9.0000.5534). Assegurou-se a não identificação das participantes e confidencialidade das informações.

\section{RESULTADOS}

Sobre os dados sociodemográficos, identificou-se que 78 (65,5\%) mulheres apresentaram idade de até 29 anos, com uma média de $27,3 \pm 7,7$ anos e variação de 18 a 45 anos. Observou-se que a maioria das participantes $(78,2 \%)$ se autodeclararam não brancas, sendo que $67(72 \%)$ indicaram cor parda. No que se refere à escolaridade, $81(68 \%)$ tinham ensino médio (10 a 12 anos de estudos), a média variou de 10,3 $\pm 2,48$ anos de estudo, com o mínimo de quatro e máximo de 14 anos de estudos. Além do mais, verificou-se que 64 (54\%) mulheres vivem com até um salário mínimo, com média variando de 1,55 \pm 0,63 salários mínimos, sendo o mínimo de 0,25 e máximo de quatro salários. Em relação aos dados reprodutivos, a maior parte, 83 (69,7\%), não possuía histórico de aborto anterior, constataram o diagnóstico da gravidez 100 (84\%), declararam gravidez não planejada 76 (64\%) e faziam uso prévio de método anticoncepcional 93 (78,2\%) (Tabela 1). Há de se destacar que 12 (10\%) mulheres, no decorrer da entrevista, relataram episódios de gravidez ou aborto repetido em menos de três meses.

Tabela 1. Características sociodemográficas e reprodutivas de mulheres internadas por aborto, em hospitais de referência. Fortaleza, Ceará, 2017

\begin{tabular}{|c|c|c|}
\hline Variável & $n=119$ & $\%$ \\
\hline \multicolumn{3}{|l|}{ Idade } \\
\hline$\leq 29$ anos & 78 & 65,5 \\
\hline$>29$ anos & 41 & 34,5 \\
\hline \multicolumn{3}{|l|}{ Cor/raça } \\
\hline Não Branca & 93 & 78,2 \\
\hline Branca & 26 & 21,8 \\
\hline \multicolumn{3}{|l|}{ Escolaridade } \\
\hline Ensino Fundamental ou menos & 38 & 32,0 \\
\hline Ensino médio/Superior & 81 & 68,0 \\
\hline
\end{tabular}


Tabela 1. Continuação...

\begin{tabular}{lcc}
\hline \multicolumn{1}{c}{ Variável } & $\mathbf{n = 1 1 9}$ & $\%$ \\
\hline Renda Familiar & 64 & 54,0 \\
\hline Até 1 salário & 55 & 46,0 \\
\hline Acima de 1 salário & & \\
\hline Número de abortos & 83 & 69,7 \\
\hline Nenhum & 36 & 30,3 \\
\hline Um ou mais abortos & & \\
\hline Diagnóstico da gravidez & 100 & $\mathbf{8 4}$ \\
\hline Teste ou Ultrassonografia & 19 & \\
\hline Não realizou teste & & 64,0 \\
\hline Gravidez planejada & 76 & 36,0 \\
\hline Não & 43 & 21,8 \\
\hline Sim & & 78,2 \\
\hline Uso de método contraceptivo prévio & 26 & \\
\hline Não & 93 & \\
\hline Sim & & \\
\hline
\end{tabular}

Com relação às condições clínicas, identificou-se que 85 (78\%) mulheres estavam com 12 semanas gestacionais ou menos no período da internação, com média de $11,7 \pm 0,7$ semanas gestacionais. Verificou-se também que 113 (95\%) mulheres referiram algum sinal e/ou sintoma que as impulsionaram para a procura do serviço de saúde. Aquelas que não relataram sinais ou sintomas foram à procura do serviço de saúde devido à revelação diagnóstica de aborto retido (Tabela 2).

Tabela 2. Características clínicas e assistenciais, antes da internação, de mulheres internadas por aborto, em hospitais de referência. Fortaleza, Ceará, 2017

\begin{tabular}{lcc}
\hline \multicolumn{1}{c}{ Variável } & $\mathbf{n = 1 1 9}$ & \% \\
\hline Idade Gestacional $^{*}$ & & \\
\hline Até 12 semanas & 25 & $\mathbf{7 8 , 0}$ \\
\hline Mais de 12 semanas & 24,0 \\
\hline Sinas e sintomas apresentados & 66 & 55,5 \\
\hline Sangramento/Dor Abdominal & 34 & 29,0 \\
\hline Sangramento & 10 & 8,0 \\
\hline Dores Abdominais & 3 & 2,5 \\
\hline Sangramento/Dores Abdominais/Febre & 6 & 5,0 \\
\hline Não Apresentou Sintomas & & \\
\hline Local em que buscou o primeiro atendimento & 104 & 87,4 \\
\hline Hospital & 5 & 4,2 \\
\hline Posto de Saúde & 10 & 8,4 \\
\hline UPA
\end{tabular}

${ }^{*} \mathrm{~N}=109$, pois 10 mulheres desconheciam a idade gestacional e data da última menstruação. ${ }^{* *} \mathrm{~N}=56$, refere-se ao número de mulheres que não foram internadas no primeiro hospital em que buscaram atendimento, tiveram que percorrer 1 ou mais hospitais para internação 
Tabela 2. Continuação...

\begin{tabular}{|c|c|c|}
\hline Variável & $n=119$ & $\%$ \\
\hline \multicolumn{3}{|c|}{ Internação no primeiro hospital em que buscou atendimento } \\
\hline Não & 56 & 47 \\
\hline Sim & 63 & 53 \\
\hline \multicolumn{3}{|l|}{ Número de hospitais percorridos** } \\
\hline 1hospital & 44 & 78,5 \\
\hline$\geq 2$ hospitais & 12 & 21,5 \\
\hline \multicolumn{3}{|l|}{ Tempo de busca para atendimento } \\
\hline Até 1 dia & 13 & 11 \\
\hline 2-5 dias & 73 & 61,3 \\
\hline$\geq 6$ dias & 28 & 23,5 \\
\hline Não Apresentou Sintomas & 5 & 4,2 \\
\hline \multicolumn{3}{|c|}{ Tempo de espera para atendimento médico } \\
\hline Menos de 60 minutos & 79 & 66,4 \\
\hline 60 minutos ou mais & 40 & 33,6 \\
\hline
\end{tabular}

${ }^{*} \mathrm{~N}=109$, pois 10 mulheres desconheciam a idade gestacional e data da última menstruação. ${ }^{* *} \mathrm{~N}=56$, refere-se ao número de mulheres que não foram internadas no primeiro hospital em que buscaram atendimento, tiveram que percorrer 1 ou mais hospitais para internação

Quanto à assistência prestada, previamente à internação, $104(87,4 \%)$ consideraram o hospital como primeiro local de busca para atendimento clínico, 56 (47\%) mulheres não tiveram admissão hospitalar na primeira instituição pretendida, $12(21,5 \%)$ destas chegaram a percorrer até dois ou mais hospitais, na busca da finalização do aborto. Sobre o intervalo de tempo entre a apresentação dos sinais e/ou sintomas e a procura de atendimento, observou-se que $86(72,3 \%)$ mulheres buscaram o serviço em até cinco dias, sendo que o mínimo de tempo foi de seis horas e o máximo foi de 21 dias, com média de 4,5 $\pm 3,6$ dias. No tocante ao tempo decorrido entre a chegada ao hospital e atendimento médico, a maioria $(66,4 \%)$ relatou a duração de menos de uma hora. O período de tempo variou de 10 minutos até 8 horas de espera para assistência médica, com média variando de $57 \pm 67,74$ minutos (Tabela 2).

Em relação à assistência hospitalar, constatou-se que, dentre as 119 mulheres internadas por abortamento, somente $74(62,2 \%)$ realizaram o exame hemograma. Quanto ao coagulograma, apenas $18(15 \%)$ dessas mulheres o fizeram e $11(9 \%)$ delas efetuaram o exame urina tipo I. Uma minoria $(33,6 \%)$ realizou o exame de imagem, ultrassonografia. Ocorreram sete diagnósticos clínicos de aborto infectado, o que correspondeu ao tratamento com antibioticoterapia. Também houve a necessidade de realização de transfusão sanguínea em três casos de hemorragia grave. Notou-se que a maioria (98,3\%) das mulheres foi submetida ao procedimento de esvaziamento uterino por curetagem para finalização do aborto. Quanto ao intervalo de tempo transcorrido entre admissão da mulher na sala de parto e a realização do procedimento cirúrgico para esvaziamento uterino, 85 (71,4\%) mulheres realizaram o procedimento em até 12 horas. $O$ período de tempo variou entre uma e 34 horas, com média de $11,07 \pm 8,18$ horas (Tabela 3 ). 
Tabela 3. Características da assistência hospitalar e de custos de mulheres internadas por aborto, em hospitais de referência. Fortaleza, Ceará, 2017

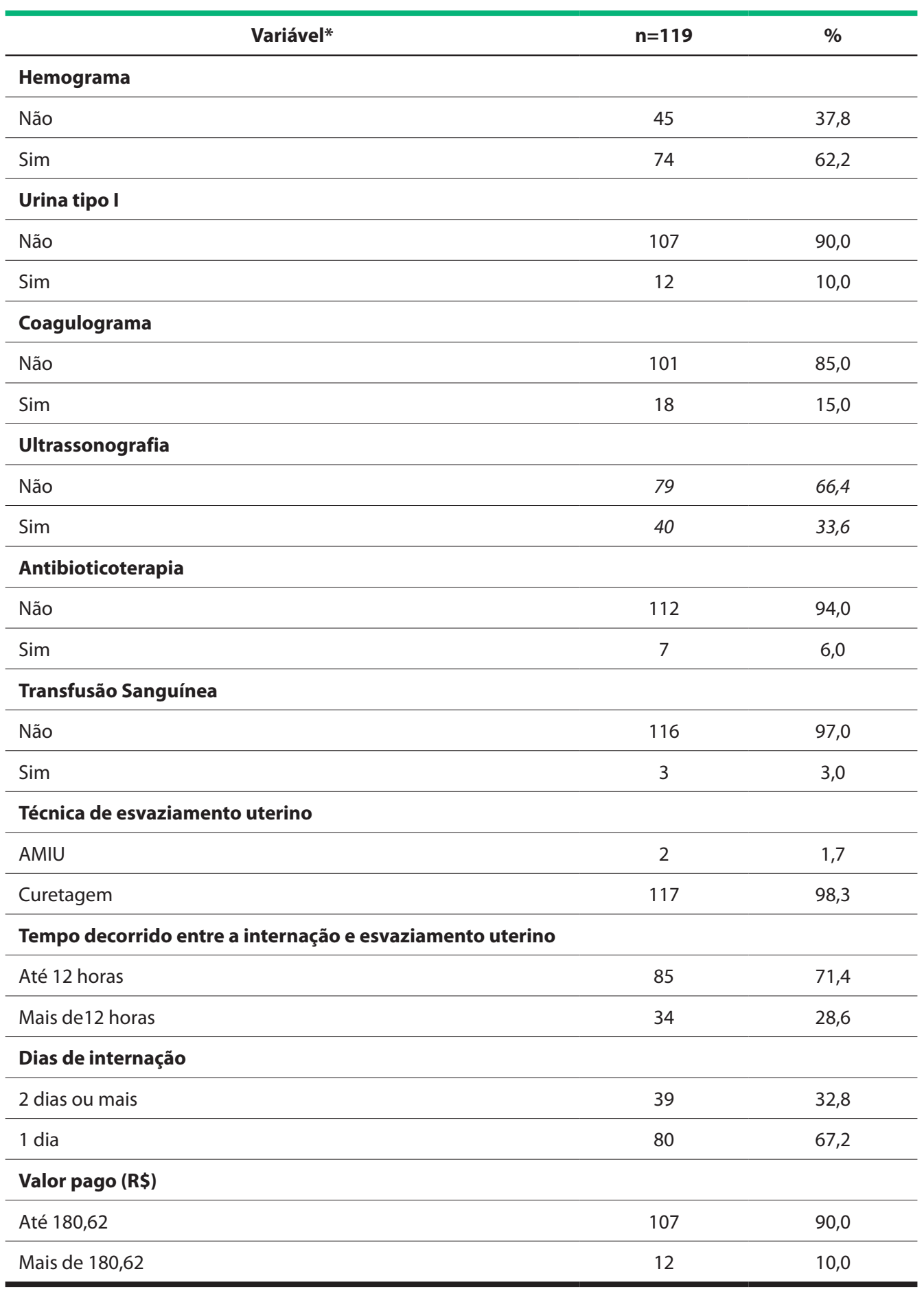

*Dados coletados nos prontuários

Sobre os custos, os resultados apontaram pluralidade de mulheres $(67,2 \%)$ que permaneceram um dia internada. O período máximo foi de sete, com média variando de $1,58 \pm 1,13$ dias de hospitalização. A maior parte $(90 \%)$ dos internamentos foi paga com valor de até $R \$ 180,62$. Sobre o valor pago, verificou-se média de $R \$ 184,89 \pm 17,00$, com pagamento mínimo de $\mathrm{R} \$ 179,62$ e máximo de $\mathrm{R} \$ 315,86$ (Tabela 3). 


\section{DISCUSSÃO}

Os resultados apontaram que a maioria das mulheres esteve na faixa etária abaixo de 30 anos, se autodeclarou não branca e de baixa renda, perfil que equivale a outros estudos ${ }^{10-12}$. Nesta pesquisa, as mulheres relataram possuir mais de oito anos de estudos; esta escolaridade foi também observada como mais frequente em outras investigações sobre mulheres em situação abortiva ${ }^{13,14}$. Notou-se que as características presentes nas participantes retratam aspectos de vulnerabilidade socioeconômica, bem como se assemelham às dos usuários que buscam atendimento nos serviços públicos de saúde.

A partir dos dados reprodutivos, observou-se elevada frequência de mulheres com primeira gestação com desfecho abortivo. Mundialmente, a população feminina vivencia uma ou mais experiências de abortamento em seu histórico obstétrico ${ }^{15}$.

Em 2016, o Ministério da Saúde publicou portaria que autorizava transferência de recursos relacionados ao Teste Rápido de Gravidez (TRG) na Unidade Básica de Saúde para reforço de sua implantação e oferta ${ }^{16}$. No entanto, das mulheres que realizaram testes confirmatórios de gravidez, a maioria declarou sua realização com recursos próprios e somente quatro delas realizaram o TRG em unidade de saúde. Este contexto traz reflexões sobre a fragilidade em um dos componentes de pré-natal da Rede Cegonha, que é a oferta doTRG para detecção precoce da gestação ${ }^{17}$, uma vez que a pesquisa demonstrou o não acesso das mulheres a este insumo.

A gestação não planejada foi a condição mais frequente; no entanto, a maioria relatou uso de anticoncepcional previamente à gravidez. Gravidez imprevista pode motivar práticas de indução ao abortamento, por vezes realizadas de forma insegura, que acarretam riscos à saúde da mulher, ou situações agravantes de morbidade ou mortalidade, principalmente em países como o Brasil, que possui lei restritiva ao aborto ${ }^{18}$.

Neste estudo, o percentual de mulheres que não relatou uso de algum método de contracepção foi semelhante aos $22,7 \%{ }^{19}$, embora bastante inferior aos $74,2 \%{ }^{20}$, evidenciados em outras pesquisas. Isto evidencia a necessidade de avaliação da efetividade e das estratégias de atenção à saúde reprodutiva, visto que a maioria das participantes teve gravidez não programada, como também relatos de gestação anterior e aborto repetido, em menos de três meses.

Com relação aos dados clínicos, os resultados apontaram alta proporção de mulheres admitidas no âmbito hospitalar com a idade gestacional menor que 12 semanas, ao comparar com outros estudos 11,13,21. A Rede Municipal de Saúde de Fortaleza está dividia em seis Secretarias Regionais de Saúde (SRS). Cada Secretaria Regional abrange Unidades de Atenção Primária à Saúde, Unidades de Pronto Atendimento e hospitais. Dentre os 24 estabelecimentos hospitalares da Rede Municipal, 12 realizam internações por abortamentos, sendo que cada SRS abrange de um a três destes hospitais. No entanto, somente a SRS IV não possui unidade hospitalar que dispõe internamento obstétrico ${ }^{22}$. Pressupõe-se que a organização da assistência ginecológica e obstétrica composta no município facilitou a busca de serviço de saúde pela mulher em situação de aborto precoce, quando ocorre antes da $13^{\text {a }}$ semana gestacional ${ }^{7}$. Quanto à assistência antes da internação, observou-se que a mulher em abortamento nem sempre é internada na primeira instituição em que procurou atendimento. Podem até transitar em dois ou mais hospitais na busca de finalizar a gravidez. Dentre as mulheres que não foram internadas no primeiro hospital de busca, identificou-se, através dos relatos e dados dos prontuários, que a maioria destas não foi devidamente referenciada e que percorreu por conta própria as instituições hospitalares na tentativa de atendimento. Mulheres em abortamento, quando sofrem retardo na assistência à saúde ou quando são referenciadas de uma unidade a outra, podem apresentar maiores complicações abortivas ${ }^{23}$.

O prazo entre as manifestações sintomáticas do aborto e a busca de atendimento apresentaram-se semelhantes a estudo realizado em Papau-Nova Guiné24. Cabe ressaltar que a procura de serviços de saúde em menos de um dia pode estar relacionada aos abortos provocados, pois mulheres que iniciam o processo do aborto clandestinamente, com uso do misoprostol em domicílio, apresentam sangramento e dores abdominais intensas, também dúvidas da efetividade da medicação. Tais fatores contribuem para que busquem atendimento nas primeiras 24 horas $^{25,26}$. A partir dos relatos das participantes, identificaram-se as seguintes 
situações para retardamento na busca dos serviços de saúde: ausência de conhecimento dos indícios das complicações abortivas, peregrinação na procura de atendimento para concluir o processo do aborto, dificuldades financeiras para o translado à Unidade e para realização de exames de imagem prescritos para diagnóstico de abortamento.

Sobre o tempo de espera para atendimento médico no âmbito hospitalar, observou-se que o acolhimento da mulher em processo abortivo define-se pela avaliação de risco, a qual se baseia na necessidade de atenção à saúde imediata ou não. Assim como em outros estudos, o tempo de espera da mulher para assistência médica variou conforme a quantidade do sangramento e da intensidade da dor manifestada ${ }^{12,27}$. Verificou-se também, a não garantia de assistência de acordo com a classificação de risco, ou seja, a não oferta de atendimento prioritário mesmo que a condição da mulher apresentasse tal urgência.

Com base na Norma Técnica da Atenção Humanizada ao Abortamento ${ }^{7}$, elencaram-se os procedimentos descritos no estudo, para observar o acompanhamento da mulher durante sua internação hospitalar. Exames laboratoriais e outras análises, quando disponíveis, devem ser realizados de acordo com as condições de risco de cada mulher em abortamento avaliada ${ }^{28}$. Contudo verificou-se que nem todas as mulheres realizaram exames para seguimento da internação, deixando de investigar outras complicações clínicas associadas à condição de abortamento.

Assim como em outros estudos, observou-se a curetagem como principal método adotado para esvaziamento uterino ${ }^{12,29}$. Também que tal procedimento é adotado independentemente da idade gestacional apresentada pela mulher. No entanto, para o manejo do aborto, a Organização Mundial da Saúde (OMS) recomenda o uso da aspiração por vácuo (manual ou elétrica) ou aborto médico, que se define pelo uso sucessivo de mifepristona e misoprostol ou somente misoprostol, na ocorrência do abortamento no primeiro trimestre gestacional (12 semas). Nos casos em que ocorra após 13 semanais gestacionais, preconiza a dilatação e evacuação (D\&E) ou aborto médico ${ }^{28}$.

O estudo também aponta que as mulheres internadas por abortamento podem esperar de 12 horas ou mais para procedimento cirúrgico para esvaziamento uterino. Constatou-se que, independentemente da situação abortiva avaliada, não são classificadas como prioridade. Desta forma, os procedimentos somente serão realizados posteriormente aos partos normais e cesarianos ${ }^{27,30}$.

No que se refere aos custos, observou-se que a permanência hospitalar atendeu à recomendação do Ministério da Saúde, em que a internação por aborto, nos hospitais conveniados pelo SUS, tenha média de estadia de até um dia $^{30}$. O valor pago pela internação de até $R \$ 180,62$ assemelha-se à quantia tabelada de $R \$ 179,62$, repassada às unidades hospitalares com convênio do SUS, na realização do procedimento curetagem pós-abortamento ${ }^{31}$. Também, identificou-se que a técnica de curetagem se sobrepôs a procedimento de aspiração manual intrauterina (AMIU), como método de escolha para esvaziamento do útero, o que representou aumento de $26 \%$ dos gastos, uma vez que o procedimento por AMIU representa o valor de R\$ 142,84, segundo a Tabela Unificada do SUS ${ }^{31}$. Vale destacar que o procedimento AMIU quando comparado à curetagem, apresenta-se mais seguro e eficaz, bem como pode proporcionar menor tempo de permanência hospitalar ${ }^{32}$.

O estudo permitiu apontar as fragilidades existentes na atenção ao abortamento, os resultados levam à constatação de falhas na rede de assistência às mulheres em situação abortiva, ausência de protocolo padrão e não acompanhamento integral durante internação hospitalar. Além do mais, verificou-se maior dispêndio no custeio dos procedimentos cirúrgicos, já que não há adoção das recomendações da OMS no manejo do aborto.

Desta forma, há necessidade de visibilizar a problemática do aborto por meio de inclusão do tema nas programações de planejamento e nas análises situacionais, para que gestores, profissionais de saúde e outros envolvidos na rede de atenção à saúde da mulher promovam políticas e estratégias para mudanças necessárias na atenção ao abortamento. 


\section{REFERÊNCIAS}

1. Departamento de Informática do Sistema Único de Saúde. Estatísticas Vitais. Óbitos de mulheres em idade fértil e óbitos maternos [Internet]. Brasília: DATASUS; 2018 [citado em 2018 mar 18]. Disponível em: http://tabnet.datasus.gov.br/cgi/deftohtm.exe?sim/cnv/pmat10uf.def

2. Monteiro MFG, Adesse L, Drezett J. Atualização das estimativas da magnitude do aborto inseguro, taxas por mil mulheres e razões por 100 nascimentos vivos do aborto inseguro por faixa etária e grandes regiões. Brasil, 1995 a 2013. Reprodução \& Climatério. 2015;30(1):11-8. http://dx.doi.org/10.1016/j.recli.2015.05.003.

3. Departamento de Informática do Sistema Único de Saúde. Morbidade Hospitalar do SUS - Por local de internação: Brasil [Internet]. Brasília: DATASUS; 2018 [citado em 2018 mar 18]. Disponível em: http:// tabnet.datasus.gov.br/cgi/deftohtm.exe?sih/cnv/niuf.def

4. Departamento de Informática do Sistema Único de Saúde. Assistência à saúde. Produções hospitalares do SUS - Por local de internação: Brasil [Internet]. Brasília: DATASUS; 2018 [citado em 2018 mar 20]. Disponível em: http://tabnet.datasus.gov.br/cgi/deftohtm.exe?sih/cnv/qiuf.def

5. Fernandes M, Gollop TR, Pedroso D, Torres JHR. Os crimes contra a vida na reforma do código penal: uma visão médico-jurista. Cienc Cult. 2012;64(2):46-8. http://dx.doi.org/10.21800/S0009-67252012000200019.

6. Wiese IRB, Saldanha AAW. Aborto inseguro na interface da saúde e do direito. Saude Soc. 2014;23(2):53647. http://dx.doi.org/10.1590/S0104-12902014000200014.

7. Brasil. Ministério da Saúde. Secretaria de Atenção à Saúde. Atenção humanizada ao abortamento: norma técnica. 2. ed. Brasília: Ministério da Saúde; 2011.

8. Aquino Estela ML, Menezes G, Barreto-de-Araújo TV, Alves MT, Alves SV, Almeida MCC et al. Qualidade da atenção ao aborto no Sistema Único de Saúde do Nordeste brasileiro: o que dizem as mulheres? Ciênc. Saúde Coletiva. 2012;17(7):1765-76.

9. Alves MTSSB, Araújo TVB, Alves SV, Marinho LFB, Schiavo E, Menezes G, et al. Atenção ao aborto no Sistema Único de Saúde no Nordeste Brasileiro: a estrutura dos serviços. Rev Bras Saúde Mater Infant. 2014;14(3):229-39. http://dx.doi.org/10.1590/S1519-38292014000300004.

10. Camargo RS, Santana DS, Cecatti JG, Pacagnella RC, Tedesco RP, Melo EF Jr, et al. Severe maternal morbidity and factors associated with the occurrence of abortion in Brazil. Int J Gynaecol Obstet. 2011;112(2):88-92. http://dx.doi.org/10.1016/j.ijgo.2010.08.013. PMid:21130447.

11. Domingos SRF, Merighi MAB, Faria ECR, Ferreira LMG. Características dos abortamentos de mulheres atendidas em uma instituição hospitalar filantrópica de Caratinga - MG. Rev. Min. Enferm. 2011;15(4):50412.

12. Adesse L, Silva KS, Bonan C, Fonseca VM. Complicações do abortamento e assistência em maternidade pública integrada ao Programa Nacional Rede Cegonha. Saúde Debate. 2015;39(106):694-706. http:// dx.doi.org/10.1590/0103-1104201510600030011.

13. Brito RCF, Gomes ALC, Ferreira ECG, Bu SMA, Souza Al. Perfil sociodemográfico e reprodutivo de mulheres com complicações do aborto em hospital no Recife. Esc Anna Nery. 2013;17(3):491-5. http://dx.doi. org/10.1590/S1414-81452013000300012.

14. Wariki WMV, Ali M, Wantania J, Kuroiwa C, Shibuya K. Post abortion care in North Sulawesi, Indonesia: Patients determinants in selection of health facility. Qual Prim Care. 2015;23(3):181-8.

15. Bonnen KI, Tuijje DN, Rasch V. Determinants of first and second trimester induced abortion - results from a cross-sectional study taken place 7 years after abortion law revisions in Ethiopia. BMC Pregnancy Childbirth. 2014;14(416):1-21. http://dx.doi.org/10.1186/s12884-014-0416-9. PMid:25622947.

16. Brasil. Ministério da Saúde. Portaria n 323, de 4 de março de 2016. Autoriza o repasse de recursos, em parcela única, para Municípios, referente aos Testes Rápidos de Gravidez do Componente Pré-Natal da Rede Cegonha. Diário Oficial da União, Brasília, 7 de março de 2016; Seção 1. p. 72-98.

17. Brasil. Ministério da Saúde. Secretaria de Atenção à Saúde. Teste rápido de gravidez na Atenção Básica: guia técnico. Brasília, DF: Ministério da Saúde; 2013.

18. Sedgh G, Singh S, Hussain R. Intended and unintended pregnancies worldwide in 2012 and recent trends. Stud Fam Plann. 2014;45(3):301-14. http://dx.doi.org/10.1111/j.1728-4465.2014.00393.x. PMid:25207494.

19. Santos TF, Andreoni S, Silva RS. Prevalência e características de mulheres com aborto provocado - Favela México 70, São Vicente - São Paulo. Rev Bras Epidemiol. 2012;15(1):123-33. PMid:22450498.

20. Santos TF, Fusco C, Silva RS. Induced and unsafe abortion 20 years after the International Conference on Population and Development, Cairo, 94: prevalence and sociodemographic characteristics. Favela México 70, São Vicente, São Paulo, Brazil. Repro Clim. 2016;31(1):5-12. 
21. Kalilani-Phiri L, Gebreselassie H, Levandowski BA, Kuchingale E, Kachale F, Kangaude L. The severity of abortion complications in Malawi. Int J Gynaecol Obstet. 2015;128(2):160-4. http://dx.doi.org/10.1016/j. ijgo.2014.08.022. PMid:25468057.

22. Fortaleza. Secretaria Municipal de Saúde. Plano Municipal de Saúde de Fortaleza: 2018-2021. Fortaleza: Secretaria Municipal de Fortaleza; 2017

23. Ziraba AK, Izugbara C, Brooke A, Levandowski BA, Gebreselassie H, Mutua M, et al. Unsafe abortion in Kenya: a cross-sectional study of abortion complication severity and associated factors. BMC Pregnancy Childbirth. 2015;15(34):1-11. http://dx.doi.org/10.1186/s12884-015-0459-6. PMid:25884662.

24. Vallely LM, Homiehombo P, Kelly-Hanku A, Kumbia A, Mola GD, Whittaker A. Hospital Admission following Induced Abortion in Eastern Highlands Province, Papua New Guinea - A Descriptive Study. PLoS One. 2014;9(10):1-10. http://dx.doi.org/10.1371/journal.pone.0110791. PMid:25329982.

25. Brasil. Ministério da Saúde. Secretaria de Ciência, Tecnologia e Insumos Estratégicos. Aborto e saúde pública no Brasil: 20 anos. Brasília: Ministério da Saúde; 2009.

26. Coast E, Murray SF. "These things are dangerous": Understanding induced abortion trajectories in urban Zambia. Soc Sci Med. 2016;153:201-9. http://dx.doi.org/10.1016/j.socscimed.2016.02.025. PMid:26921835.

27. Souza CHD, Porto RM, Carvalho DRGS. Paradoxos da atenção ao aborto provocado: "urgências" e "emergências" em uma Maternidade Escola em Natal/RN. Bagoas. 2013;7(9):43-69.

28. Organización Mundial de la Salud. Manual de práctica clínica para un aborto seguro. Geneva:OMS; 2014.

29. Ikeako L, Onoh R, Ezegwui H, Ezeonu P. Pattern and Outcome of Induced Abortion in Abakaliki, Southeast of Nigeria. Ann Med Health Sci Res. 2014;4(3):442-6. http://dx.doi.org/10.4103/2141-9248.133475. PMid:24971223.

30. Mccallum C, Menezes G, Reis AP. O dilema de uma prática: experiências de aborto em uma maternidade pública de Salvador, Bahia. Hist Cienc Saude Manguinhos. 2016;23(1):37-56. http://dx.doi.org/10.1590/ S0104-59702016000100004. PMid:27008073.

31. Sistema de Gerenciamento da Tabela de Procedimentos. Medicamentos e OPMS do SUS. Consultar Procedimentos - Brasil [Internet] Brasília: SIGTAP; 2018 [citado 2018 mar 18]. Disponível em: http://sigtap. datasus.gov.br/tabela-unificada/app/sec/inicio.jsp

32. Saciloto MP, Konopka CK, Velho MTC, Jobim FC, Resener EV, Muradás RR, et al. Aspiração manual intrauterina no tratamento do abortamento incompleto até 12 semanas gestacionais: uma alternativa à curetagem uterina. Rev Bras Ginecol Obstet. 2011;33(10):292-6. PMid:22231162. 